\title{
ON THE RELATIONSHIP OF AP, RS AND CEP IN CONGRUENCE MODULAR VARIETIES. II
}

\author{
CLIFFORD BERGMAN AND RALPH MCKENZIE
}

(Communicated by Donald Passman)

\begin{abstract}
Let $V$ be a congruence distributive variety, or a congruence modular variety whose free algebra on 2 generators is finite. If $V$ is residually small and has the amalgamation property, then it has the congruence extension property. Several applications are presented.
\end{abstract}

In two previous papers [1 and 2], we considered the following question: if $\mathscr{V}$ is a residually small variety with the amalgamation property, must $\mathscr{V}$ have the congruence extension property? Our work established the following implications for a congruence modular variety $\mathscr{V}$ :

(1) If $\mathscr{V}$ is 2-finite and has $\mathrm{C} 2$, then $\mathrm{AP}+\mathrm{RS} \Rightarrow \mathrm{R}$.

(2) If $\mathscr{V}$ is 4-finite with $\mathrm{C} 2$ and $\mathrm{R}$, then $\mathrm{AP}+\mathrm{RS} \Rightarrow \mathrm{CEP}$.

(The terminology will be explained below.)

In this paper we supplement and extend these results. Assuming still that $\mathscr{V}$ is congruence modular, we have:

(3) $\mathrm{AP}+\mathrm{RS} \Rightarrow \mathrm{C} 2$.

(4) If $\mathscr{V}$ has $\mathrm{R}$, then AP $+\mathrm{RS} \Rightarrow \mathrm{CEP}$.

Combining these implications, we have that every congruence modular, 2-finite variety satisfies $\mathrm{AP}+\mathrm{RS} \Rightarrow \mathrm{CEP}$. Furthermore, every congruence distributive variety (no finiteness assumption) satisfies $\mathrm{AP}+\mathrm{RS} \Rightarrow \mathrm{CEP}$.

Our universal algebraic notation and terminology are standard. Good references are $[\mathbf{4}$ and 9$]$.

Let $\mathscr{V}$ be a variety of algebras. We say that $\mathscr{V}$

- has the amalgamation property (AP) if, for all $\mathbf{A}, \mathbf{B}_{0}, \mathbf{B}_{1} \in \mathscr{V}$ and all embeddings $f_{i}: \mathbf{A} \rightarrow \mathbf{B}_{i}$, for $i=0,1$, there is $\mathbf{C} \in \mathscr{V}$ and embeddings $g_{i}: \mathbf{B}_{i} \rightarrow \mathbf{C}, i=0,1$, such that $g_{0} \circ f_{0}=g_{1} \circ f_{1}$,

- is residually small (RS) if there is a cardinal $\kappa$ such that every subdirectly irreducible algebra in $\mathscr{V}$ has cardinality less than $\kappa$,

- has the congruence extension property (CEP) if, for all $\mathbf{A} \leq \mathbf{B} \in \mathscr{V}$, and congruence $\alpha$ on $\mathbf{A}$, there is $\bar{\alpha} \in$ Con $\mathbf{B}$ such that $\bar{\alpha} \uparrow \mathbf{A}=\alpha$,

- is $n$-finite, for a positive integer $n$, if every member of $\mathscr{V}$ generated by $n$ elements is finite.

Received by the editors December 7, 1987.

1980 Mathematics Subject Classification (1985 Revision). Primary 08B10, 08B25; Secondary 03C25, $20 \mathrm{E} 06$.

Research partially supported by the Iowa State University Sciences and Humanities Research Institute and National Science Foundation Grant No. DMS-8600300.

(C) 1988 American Mathematical Society $0002-9939 / 88 \$ 1.00+\$ .25$ per page 
The least nontrivial congruence on a subdirectly irreducible algebra is called the monolith, and the least and greatest congruences on an algebra are denoted 0 and 1 respectively. Let $\mathbf{B}$ be a subalgebra of $\mathbf{A}_{0} \times \mathbf{A}_{1}$. It is often convenient to denote the elements of $\mathbf{B}$ as vertical pairs $\left(\begin{array}{l}x_{0} \\ x_{1}\end{array}\right)$ with $x_{i} \in A_{i}$ for $i=0,1$. Let $\alpha \in \operatorname{Con} \mathbf{A}_{0}$. Then there is an induced congruence $\alpha_{0}$ on $\mathbf{B}$ given by $\left(\begin{array}{l}x_{0} \\ x_{1}\end{array}\right) \alpha_{0}\left(\begin{array}{l}y_{0} \\ y_{1}\end{array}\right) \Leftrightarrow x_{0} \alpha y_{0}$. Similarly, for $\beta \in$ Con $\mathbf{A}_{1}$, there is a congruence $\beta_{1}$ on $\mathbf{B}$ defined in an analogous way. The particularly important congruences $0_{0}$ and $0_{1}$ are denoted $\eta_{0}$ and $\eta_{1}$ respectively. Given a congruence $\beta$ on an algebra $\mathbf{D}$, we write $\mathbf{D}(\beta)$ for the subalgebra of $\mathbf{D}^{2}$ with universe $\beta$. That is: $D(\beta)=\left\{\left(\begin{array}{l}x \\ y\end{array}\right): x \beta y\right\}$.

The commutator is a binary operation, denoted [,], defined on the congruence lattice of an algebra. In a congruence modular variety $\mathscr{V}$, the commutator has some strong properties. It is additive, that is

$$
[\alpha, \bigvee \Theta]=\bigvee_{\theta \in \Theta}[\alpha, \theta] \quad \text { for } \mathbf{A} \in \mathscr{V}, \alpha \in \operatorname{Con} \mathbf{A}, \text { and } \Theta \subseteq \operatorname{Con} \mathbf{A}
$$

symmetric and meet-dominated

$$
[\alpha, \beta]=[\beta, \alpha] \subseteq \alpha \wedge \beta \quad \text { for } \mathbf{A} \in \mathscr{V} \text { and } \alpha, \beta \in \operatorname{Con} \mathbf{A},
$$

respects finite direct products

$$
\begin{aligned}
& \text { for } \alpha, \beta \in \text { Con } \mathbf{A} \text { and } \gamma, \delta \in \operatorname{Con} \mathbf{B} \\
& {\left[\alpha_{0} \wedge \gamma_{1}, \beta_{0} \wedge \delta_{1}\right]=[\alpha, \beta]_{0} \wedge[\gamma, \delta]_{1} \text { in } \mathbf{A} \times \mathbf{B},}
\end{aligned}
$$

and exhibits the following homomorphism property

$$
\begin{aligned}
& \text { if } f: \mathbf{A} \rightarrow \mathbf{B} \text { is a surjective homomorphism and } \alpha, \beta \in \text { Con } \mathbf{B} \text { then } \\
& f^{-1}[\alpha, \beta]=\left[f^{-1} \alpha, f^{-1} \beta\right] \vee \operatorname{ker} f .
\end{aligned}
$$

For a systematic development of the subject, we direct the reader to [6], especially Chapter 4 .

There are three identities involving the commutator that we consider in this paper. We say that an algebra $\mathbf{A}$ has:

$$
\begin{array}{llll}
\mathrm{C} 1 & \text { if } & \forall \alpha, \beta \in \mathrm{Con} \mathbf{A} & \alpha \wedge[\beta, \beta]=[\alpha \wedge \beta, \beta] \\
\mathrm{C} 2 & \text { if } & \forall \alpha, \beta \in \mathrm{Con} \mathbf{A} & {[\alpha, \beta]=\alpha \wedge \beta \wedge[1,1]} \\
\mathrm{R} & \text { if } & \forall \mathbf{B} \leq \mathbf{A} & {\left[1_{\mathbf{A}}, 1_{\mathbf{A}}\right]\left\lceil\mathbf{B}=\left[1_{\mathbf{B}}, 1_{\mathbf{B}}\right]\right.}
\end{array}
$$

We say that a class $\mathscr{K}$ has one of these properties if and only if every member of $\mathscr{K}$ has the property. Finally for congruences $\alpha, \beta$ on an algebra $\mathbf{A}$, we define $(\alpha: \beta)$ to be the largest congruence $\theta$ such that $[\beta, \theta] \leq \alpha$.

We collect some facts connecting these concepts in the following theorem.

THEOREM 0. Let $\mathscr{V}$ be a congruence modular variety.

(1) $\mathscr{V}$ satisfies $\mathrm{C} 1$ if and only if, for every subdirectly irreducible algebra $\mathbf{A}$ of $\mathscr{V}$ with monolith $\beta$, if $\theta=(0: \beta)$ then $[\theta, \theta]=0$.

(2) $\mathscr{V}$ satisfies $\mathrm{C} 2$ if and only if every subdirectly irreducible algebra $\mathbf{A}$ of $\mathscr{V}$ satisfies $\mathrm{C} 2$, and this in turn is equivalent to $\mathbf{A}$ being either abelian or prime.

(3) $\mathrm{C} 2 \Rightarrow \mathrm{C} 1$. 
(4) If $\mathscr{V}$ is a residually small variety then it satisfies $\mathrm{C} 1$. Conversely, if $\mathscr{V}$ is finitely generated and satisfies $\mathrm{C} 1$, then it is residually small.

(5) Every variety with $C E P$ satisfies $\mathrm{C} 2+R$.

(1) and (4) are from [5], (2) and (5) are from [7]. (A is prime iff $\alpha, \beta \neq 0 \Rightarrow$ $[\alpha, \beta] \neq 0$.) (3) follows easily from the definitions.

Our first objective is the following

THEOREM 1. Let $\mathscr{V}$ be a congruence modular variety. If $\mathscr{V}$ has $\mathrm{AP}$ and $\mathrm{RS}$, then $\mathscr{V}$ has $\mathrm{C} 2$.

We require a pair of lemmas. The first of these is virtually identical to Theorem 10.9 of [6]. We include the proof for completeness.

LEMMA 2. Let $\mathscr{V}$ be a congruence modular, residually small variety. If $\mathscr{V}$ fails $\mathrm{C} 2$, then $\mathscr{V}$ contains a subdirectly irreducible algebra $\mathbf{A}$ with monolith $\beta$, and an endomorphism $f$ such that

(1) $0=[\beta, \beta]<\beta=[\beta, 1]$

(2) $f=f^{2}$

(3) $x \beta y \Leftrightarrow f(x)=f(y) \beta y$.

ProOF. By Theorem $0, \mathscr{V}$ satisfies $\mathrm{C} 1$ since it is residually small. If $\mathscr{V}$ fails $\mathrm{C} 2$, there is a subdirectly irreducible algebra $\mathbf{D}$ which is neither abelian nor prime. Let $\gamma$ be the monolith of $\mathbf{D}$ and $\kappa=(0: \gamma)$. Then, since $\mathbf{D}$ is not prime, $[\gamma, \gamma]=0$ and since $\mathbf{D}$ is not abelian, but satisfies $\mathrm{C} 1, \gamma \leq \kappa<1$ and $[\kappa, \kappa]=0$.

Let $\Delta=\Delta_{\gamma, \kappa}$ be the congruence on $\mathbf{D}(\gamma)$ generated by

$$
\left\{\left\langle\left(\begin{array}{l}
x \\
x
\end{array}\right),\left(\begin{array}{l}
y \\
y
\end{array}\right)\right\rangle: x \kappa y\right\} \text {. }
$$

Observe that on $\mathbf{D}(\gamma), \kappa_{i}=\left(\eta_{i}: \gamma_{i}\right)$, for $i=0,1$, since

$$
\left[\delta, \gamma_{i}\right] \leq \eta_{i} \Rightarrow\left[\delta \vee \eta_{i}, \gamma_{i}\right]=\left[\delta, \gamma_{i}\right] \vee\left[\eta_{i}, \gamma_{i}\right] \leq \eta_{i} \Rightarrow \delta \leq \delta \vee \eta_{i} \leq \kappa_{i}
$$

by the homomorphism property of the commutator. Furthermore the following relationships hold among the congruences of $\mathbf{D}(\gamma)$ :

$$
\begin{gathered}
\eta_{i} \prec \gamma_{i} \leq \kappa_{i} \\
\gamma_{0}=\gamma_{1}=\eta_{0} \vee \eta_{1} \quad \text { for } i=0,1 . \\
\Delta \vee \eta_{i}=\kappa_{0}=\kappa_{1} \\
\Delta \wedge \eta_{i}=0
\end{gathered}
$$

The last of these follows from the fact that $[\gamma, \kappa]=0$. See [6, Theorem 4.9]. It follows from these identities that $\gamma_{0} / \eta_{0} \searrow \eta_{1} / 0 \nearrow \kappa_{0} / \Delta \searrow \eta_{0} / 0 \nearrow \gamma_{1} / \eta_{1}$, and therefore, $\eta_{0}$ and $\eta_{1}$ are atoms of $\operatorname{Con} \mathbf{D}(\gamma)$.

We claim that $\Delta$ is a completely meet-irreducible congruence with (unique) cover $\kappa_{0}$. Let $\lambda>\Delta$ and $\lambda \neq \kappa_{0}$. From the computation above, $\kappa_{0}$ covers $\Delta$, so $\lambda \Varangle \kappa_{0}$. Since $\kappa_{0}=\left(\eta_{0}: \gamma_{0}\right)$ we have $\left[\lambda, \eta_{0} \vee \eta_{1}\right]=\left[\lambda, \gamma_{0}\right] \not \eta_{0}$ and therefore $\left[\lambda, \eta_{1}\right] \not \eta_{0}$. It follows that $\lambda \wedge \eta_{1} \neq 0$. But $\eta_{1}$ is an atom, so $\lambda \geq \eta_{1}$, thus $\lambda \geq \eta_{1} \vee \Delta=\kappa_{0}$ as desired.

Define $\mathbf{A}$ to be $\mathbf{D}(\gamma) / \Delta$. Then $\mathbf{A}$ is subdirectly irreducible with monolith $\beta=$ $\kappa_{0} / \Delta$. Note that $\left[\kappa_{0}, \kappa_{0}\right]=\left[\eta_{0} \vee \Delta, \eta_{1} \vee \Delta\right] \leq \Delta$, so $[\beta, \beta]=0$ on $\mathbf{A}$. Also $\mathbf{A}$ is 
nonabelian. For if it were abelian, then in $\mathbf{D}(\gamma),\left[\eta_{1}, 1\right] \leq \Delta \wedge \eta_{1}=0$. But then $\left[\gamma_{0}, 1\right]=\left[\eta_{0} \vee \eta_{1}, 1\right] \leq \eta_{0}$ which contradicts the fact that $\left(\eta_{0}: \gamma_{0}\right)=\kappa_{0}<1$. Therefore, in $\mathbf{A},[\beta, 1]=\beta$, by $\mathrm{C} 1$, verifying (1) of the lemma.

We define $f: \mathbf{A} \rightarrow \mathbf{A}$ by $f\left(\left(\begin{array}{l}x \\ y\end{array}\right) / \Delta\right)=\left(\begin{array}{l}x \\ x\end{array}\right) / \Delta$. As $\Delta \leq \kappa_{0}$, this is well defined. That $f$ is an endomorphism satisfying conditions (2) and (3) above is a straightforward verification.

In order to continue, we need to recall some facts about our modular variety $\mathscr{V}$. There is a ternary term $d$ in the language of $\mathscr{V}$, called the difference term, with the following properties:

(1) $\mathscr{V} \vDash d(x, x, y)=y$.

(2) For every $\mathbf{B}$ in $\mathscr{V}$, abelian congruence $\theta$ on $\mathbf{B}$, and $b \in B,\langle b / \theta, d\rangle$ is a ternary group, denoted $M(\theta, b)$, and for each $n$-ary term function $t$, if $t\left(b_{1}, b_{2}, \ldots, b_{n}\right)=c$ then $t$ is a ternary group homomorphism from $M\left(\theta, b_{1}\right) \times \cdots \times M\left(\theta, b_{n}\right)$ to $M(\theta, c)$.

For the appropriate definitions and proofs see [6, 5.5-5.8].

LEMMA 3. Let $\mathbf{A}$ be an algebra satisfying the conclusions of Lemma 2. There are automorphisms $e_{0}$ and $e_{1}$ of $\mathbf{A}(\beta)$ given by:

$$
e_{0}\left(\begin{array}{l}
x \\
y
\end{array}\right)=\left(\begin{array}{c}
d(x, f x, y) \\
y
\end{array}\right) \quad \text { and } \quad e_{1}\left(\begin{array}{l}
x \\
y
\end{array}\right)=\left(\begin{array}{c}
x \\
d(x, f y, y)
\end{array}\right)
$$

where $d$ is the difference term for $\mathscr{V}$.

Proof. Recall that for any $x, y \in A,\left(\begin{array}{l}x \\ y\end{array}\right) \in A(\beta) \Leftrightarrow x \beta y \Leftrightarrow f(x)=f(y) \beta y$ by (3) of Lemma 2. Suppose $e_{0}\left(\begin{array}{l}x \\ y\end{array}\right)=e_{0}\left(\begin{array}{l}u \\ v\end{array}\right)$. Then $y=v$ and therefore $x \beta u$, so $f(x)=f(u)$ and all six elements lie in the same ternary group, $M(\beta, x)$. We have $x-f(x)+y=d(x, f(x), y)=d(u, f(u), v)=u-f(x)+y$, so $x=u$. Thus $e_{0}$ is injective. Similarly, one can check that

$$
e_{0}^{-1}\left(\begin{array}{l}
x \\
y
\end{array}\right)=\left(\begin{array}{c}
d(x, y, f x) \\
y
\end{array}\right)
$$

That $e_{0}$ is a homomorphism follows from the fact that every term, in particular every basic operation, is a ternary group homomorphism on the $M(\beta, x)$-blocks.

Proof of TheOrem 1. Assume that $\mathscr{V}$ has AP and RS, but fails C2. We shall derive a contradiction. Let $\mathbf{A}, \beta, e_{0}$ and $e_{1}$ be as in Lemmas 2 and 3. Since $\mathscr{V}$ is residually small, there is a maximal essential extension, $\mathbf{E}$, of $\mathbf{A}$ in $\mathscr{V}$ (see [12]). Observe that $\mathbf{E}$ is subdirectly irreducible. Call its monolith $\mu$. Without loss of generality, we may assume that $\mathbf{A} \subseteq \mathbf{E}$ and $\mu \uparrow A \supseteq \beta$.

The automorphisms $e_{0}$ and $e_{1}$ of Lemma 3 are also embeddings of $\mathbf{A}(\beta)$ into $\mathbf{E}^{2}$. Let us also define $e_{2}: \mathbf{A}(\beta) \rightarrow \mathbf{E}^{2}$ to be the identity map. By the amalgamation property (applied twice), there is an algebra $\mathbf{Q}$ in $\mathscr{V}$ and maps $s_{j}: \mathbf{E}^{2} \rightarrow \mathbf{Q}$, for $j=0,1,2$, such that $s_{0} \circ e_{0}=s_{1} \circ e_{1}=s_{2} \circ e_{2}$. Furthermore, there is a map $r: \mathbf{E} \rightarrow \mathbf{E}^{2}$ given by $r(x)=\left(\begin{array}{l}x \\ x\end{array}\right)$. Then $r\lceil A: \mathbf{A} \rightarrow \mathbf{A}(\beta)$, and Figure 1 commutes.

Since $\mathbf{E}$ is a maximal essential extension of $\mathbf{A}$, it is an absolute retract in $\mathscr{V}$, that is, a retract of each of its $\mathscr{V}$-extensions. Therefore there is a retraction $u: \mathbf{Q} \rightarrow \mathbf{E}$ such that $u \circ s_{2} \circ r=\mathrm{id}_{\mathbf{E}}$. Define

$$
\rho_{i}=\operatorname{ker}\left(u \circ s_{i}\right) \in \operatorname{Con} \mathbf{E}^{2} \quad \text { for } i=0,1,2 .
$$




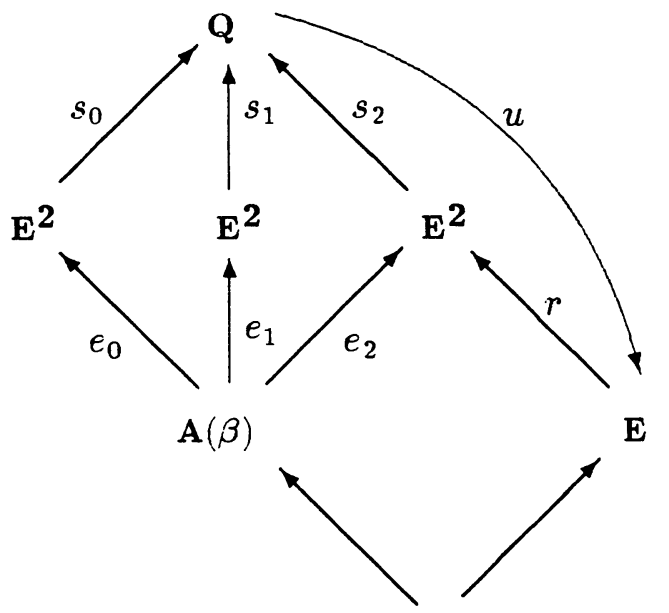

A

FIGURE 1

ClAIM. For each $i$, if $\rho_{i} \neq 0$, then either

$$
\begin{aligned}
& \rho_{i} \geq \eta_{0} \wedge \mu_{1} \text { and } \rho_{i} \wedge \eta_{1}=0 \text { or } \\
& \rho_{i} \geq \eta_{1} \wedge \mu_{0} \text { and } \rho_{i} \wedge \eta_{0}=0 .
\end{aligned}
$$

PROOF OF CLAIM. Let us write $\rho$ for $\rho_{i}$ in the proof of the claim. For any nonzero congruence $\alpha$ on $\mathbf{E},\left[\mathbf{1}_{\mathbf{E}}, \alpha\right] \supseteq\left[1_{\mathbf{E}}, \mu\right] \supseteq\left[\mathbf{1}_{\mathbf{A}}, \beta\right]=\beta \neq 0_{\mathbf{A}}$; that is, $\mathbf{E}$ is centerless. Therefore, by the homomorphism property, $\mathbf{E}^{2}$ is centerless. Thus

$$
0_{\mathbf{E}^{2}} \neq[1, \rho]=\left[\eta_{0} \vee \eta_{1}, \rho\right]=\left[\eta_{0}, \rho\right] \vee\left[\eta_{1}, \rho\right]
$$

so, say $\left[\eta_{0}, \rho\right] \neq 0$. Then $\eta_{0} \wedge \rho \neq 0$, implying $\rho \geq \eta_{0} \wedge \mu_{1}$ (since $\eta_{0} / 0 \nearrow 1 / \eta_{1}$ ). Furthermore, $\rho \wedge \eta_{1}=0$, for if not, then $\rho \geq \eta_{1} \wedge \mu_{0}$, so $\rho \geq\left(\eta_{0} \wedge \mu_{1}\right) \vee\left(\eta_{1} \wedge \mu_{0}\right)=$ $\mu_{0} \wedge \mu_{1}$. But choose $(a, b) \in \beta-0_{\mathbf{A}}$. Recall $\mu \supseteq \beta$ and $f(a)=f(b)$. Since $d$ is a term, it follows that $d(a, f a, a) \mu d(b, f b, b)$ and therefore $e_{i}\left(\begin{array}{l}a \\ a\end{array}\right) \equiv e_{i}\left(\begin{array}{l}b \\ b\end{array}\right)$ $\left(\bmod \mu_{0} \wedge \mu_{1}\right)$, hence modulo $\rho$ as well. But then $u \circ s_{i} \circ e_{i}\left(\begin{array}{l}a \\ a\end{array}\right)=u \circ s_{i} \circ e_{i}\left(\begin{array}{l}b \\ b\end{array}\right)$, so by the commutativity of Figure 1, $a=u \circ s_{2} \circ r(a)=u \circ s_{2} \circ r(b)=b$, which is a contradiction. Therefore we must have $\rho \nsupseteq \eta_{1} \wedge \mu_{0}$, so $\rho \wedge \eta_{1}=0$. This proves the claim.

We first apply the claim to $\rho_{2}$. Certainly, $\rho_{2} \neq 0$, in fact, $\left(\begin{array}{l}x \\ y\end{array}\right) \rho_{2}\left(\begin{array}{l}z \\ z\end{array}\right)$, for $z=u \circ s_{2}\left(\begin{array}{l}x \\ y\end{array}\right)$ (since $u \circ s_{2}\left(\begin{array}{l}z \\ z\end{array}\right)=u \circ s_{2} \circ r(z)=z$.) Let us assume that $\rho_{2} \geq \eta_{0} \wedge \mu_{1}$, and we will derive a contradiction. The case $\rho_{2} \geq \eta_{1} \wedge \mu_{0}$ will follow by symmetry. We have $\left[1, \rho_{2}\right]=\left[\eta_{0} \vee \eta_{1}, \rho_{2}\right] \leq \eta_{0} \vee\left(\eta_{1} \wedge \rho_{2}\right)=\eta_{0}$, so $\left[1, \rho_{2} \vee \eta_{0}\right] \leq \eta_{0}$ implying $\rho_{2} \leq \eta_{0}$ since $\mathbf{E}$ is centerless. For any $x, y \in E,\left(\begin{array}{l}x \\ y\end{array}\right) \equiv r \circ u \circ s_{2}\left(\begin{array}{l}x \\ y\end{array}\right)=\left(\begin{array}{l}z \\ z\end{array}\right)$ $\left(\bmod \rho_{2}\right)$ for some $z \in E$, whence $x=z$. Thus $\left(\begin{array}{l}x \\ y\end{array}\right) \rho_{2}\left(\begin{array}{l}x \\ x\end{array}\right) \rho_{2}\left(\begin{array}{l}x \\ y^{\prime}\end{array}\right)$ and we conclude that $\rho_{2}=\eta_{0}$.

Therefore

$$
\eta_{0}^{\mathbf{A}(\beta)}=e_{2}^{-1}\left(\rho_{2}\right)=\operatorname{ker}\left(u \circ s_{2} \circ e_{2}\right)=\operatorname{ker}\left(u \circ s_{0} \circ e_{0}\right)=e_{0}^{-1}\left(\rho_{0}\right)
$$


Observe that for every $a \in A, e_{0}\left(\begin{array}{c}f a \\ a\end{array}\right)=\left(\begin{array}{c}d(f a, f a, a) \\ a\end{array}\right)=\left(\begin{array}{l}a \\ a\end{array}\right)$. Choose $(a, b) \in \beta-0_{\mathbf{A}}$. Then

$$
\left(\begin{array}{c}
f a \\
a
\end{array}\right) \eta_{0}\left(\begin{array}{c}
f b \\
b
\end{array}\right) \Rightarrow u \circ s_{0} \circ e_{0}\left(\begin{array}{c}
f a \\
a
\end{array}\right)=u \circ s_{0} \circ e_{0}\left(\begin{array}{c}
f b \\
b
\end{array}\right) \Rightarrow\left(\begin{array}{l}
a \\
a
\end{array}\right) \rho_{0}\left(\begin{array}{l}
b \\
b
\end{array}\right) .
$$

Now apply the claim to $\rho_{0}$. If $\rho_{0} \geq\left(\eta_{0} \wedge \mu_{1}\right)$ then $\left(\begin{array}{l}a \\ b\end{array}\right) \rho_{0}\left(\begin{array}{l}a \\ a\end{array}\right) \rho_{0}\left(\begin{array}{l}b \\ b\end{array}\right)$, contradicting $\rho_{0} \wedge \eta_{1}=0$. If $\rho_{0} \geq\left(\eta_{1} \wedge \mu_{0}\right)$ then

$$
\eta_{0}=e_{0}^{-1}\left(\rho_{0}\right) \geq e_{0}^{-1}\left(\eta_{1} \wedge \mu_{0}\right) \geq \eta_{1} \wedge \beta_{0}
$$

on $\mathbf{A}(\beta)$ which is false, proving the theorem.

We now turn to our second theorem. In [1, Theorem 6] it was proved that if $\mathscr{V}$ is congruence modular, 4-finite and satisfies $\mathrm{C} 2$ and $\mathrm{R}$, then $\mathscr{V}$ satisfies $\mathrm{AP}+\mathrm{RS} \Rightarrow \mathrm{CEP}$. Theorem 1 above eliminates the need to assume $\mathrm{C} 2$. The proof below does without the assumption of 4-finiteness. In addition, it provides a considerable simplification of the previous argument. We require one lemma from that paper.

LEMMA. Let $\mathscr{V}$ be a congruence modular variety satisfying $A P$ and $R S$. Let $\mathbf{A}$ be a subdirectly irreducible member of $\mathscr{V}$, and assume $\mathbf{A}$ is an essential extension of $\mathbf{B}_{0} \times \mathbf{B}_{1}$. Then either $\mathbf{B}_{0}$ or $\mathbf{B}_{1}$ is trivial.

THEOREM 4. Let $\mathscr{V}$ be congruence modular and satisfy $R$. If $\mathscr{V}$ has AP and $R S$, then $\mathscr{V}$ has $C E P$.

Proof. By Theorem 1, $\mathscr{V}$ has C2. Let $\mathbf{A}, \mathbf{B} \in \mathscr{V}$, with $\mathbf{A} \leq \mathbf{B}$ and $\theta$ a completely meet-irreducible congruence on $\mathbf{A}$. It suffices to show that $\theta$ extends to B. By $R,\left[\mathbf{1}_{\mathbf{B}}, 1_{\mathbf{B}}\right]\left\lceil\mathbf{A}=\left[\mathbf{1}_{\mathbf{A}}, \mathbf{1}_{\mathbf{A}}\right]\right.$. Thus $\mathbf{A} /[1,1]$ can be embedded in $\mathbf{B} /[1,1]$. Suppose first that $\theta \geq[1,1]$ on $\mathbf{A}$. Since $\mathbf{B} /[1,1]$ is abelian, it has CEP, so the congruence $\theta /[1,1]$ (of $\mathbf{A} /[1,1]$ ) extends to a congruence $\psi /[1,1]$ on $\mathbf{B} /[1,1]$. Then $\psi \in$ Con $\mathbf{B}$ and $\psi \uparrow \mathbf{A}=\theta$.

So we may assume that $\theta \nsucceq[1,1]$. Let $f$ be the embedding of $\mathbf{A}$ into $\mathbf{B}$. Define $\mathbf{S}$ to be $(\mathbf{A} / \theta) \times \mathbf{A}$ and $g: \mathbf{A} \rightarrow \mathbf{S}$ by $g(a)=\left(\begin{array}{c}a / \theta \\ a\end{array}\right)$. We apply the amalgamation property to $(\mathbf{A}, \mathbf{B}, \mathbf{S}, f, g)$ yielding $(\mathbf{D}, \bar{f}, \bar{g})$ with $\mathbf{D} \in \mathscr{V}$ (Figure 2).

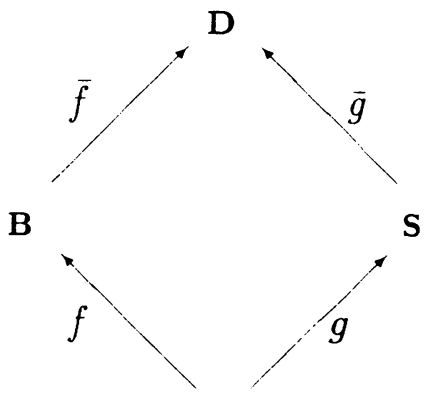

A 
Let $\eta_{0}$ and $\eta_{1}$ denote the coordinate projection kernels on $\mathbf{S}$. It suffices to find $\gamma$ in Con $\mathbf{D}$ such that $\bar{g}^{-1}(\gamma)=\eta_{0}$. By the assumptions on $\theta, \mathbf{A} / \theta$ is subdirectly irreducible with monolith $\mu$. Thus, there is a congruence $\mu_{0}$ on $\mathbf{S}$ covering $\eta_{0}$. The commutator respects finite direct products, so $\left[1_{\mathbf{S}}, 1_{\mathbf{S}}\right]=[1,1]_{0} \wedge[1,1]_{1} \geq \mu_{0} \wedge \eta_{1}$ since $\mathbf{A} / \theta$ is nonabelian. Therefore

$$
\text { for all } \beta \in \text { Con } \mathbf{S} \quad \beta \not \eta_{0} \Leftrightarrow \beta \geq \mu_{0} \wedge \eta_{1} \text {. }
$$

PROOF. Certainly, $\eta_{0} \geq \beta \geq \mu_{0} \wedge \eta_{1}$ implies $\mu_{0} \wedge \eta_{1}=\mu_{0} \wedge \eta_{1} \wedge \eta_{0}=0$, which is false. Conversely, if $\beta \npreceq \eta_{0}$ then $\beta \vee \eta_{0} \geq \mu_{0}$. Therefore by additivity and C2,

$$
\beta \geq\left[\beta \vee \eta_{0}, \beta \vee \eta_{1}\right]=\left(\beta \vee \eta_{0}\right) \wedge\left(\beta \vee \eta_{1}\right) \wedge[1,1] \geq \mu_{0} \wedge \eta_{1}
$$

Now let $\gamma$ be a maximal congruence on $\mathbf{D}$ such that $\bar{g}^{-1}(\gamma) \leq \eta_{0}^{\mathbf{S}}$. Then $\gamma$ is completely meet-irreducible. For suppose $\gamma=\bigcap_{j \in J} \gamma_{j}$ with $\gamma_{j}>\gamma$, all $j$. Then $\bar{g}^{-1}\left(\gamma_{j}\right) \not \eta_{0}$, so by $(*), \bar{g}^{-1}\left(\gamma_{j}\right) \geq \mu_{0} \wedge \eta_{1}$, for all $j \in J$, and therefore

$$
\bar{g}^{-1}(\gamma)=\bigcap_{j \in J} \bar{g}^{-1}\left(\gamma_{j}\right) \geq \mu_{0} \wedge \eta_{1}
$$

which implies

$$
g^{-1}(\gamma) \nless \eta_{0},
$$

which is a contradiction.

Therefore $\mathbf{D} / \gamma$ is subdirectly irreducible and $\bar{g}^{-1}(\gamma)=\eta_{0} \wedge \delta_{1}$ for some $\delta \in$ Con $\mathbf{A}$, by modularity. Then $\mathbf{S} / \bar{g}^{-1}(\gamma) \cong \mathbf{A} / \theta \times \mathbf{A} / \delta$ and we have an induced embedding $\bar{g} / \gamma: \mathbf{A} / \theta \times \mathbf{A} / \delta \rightarrow \mathbf{D} / \gamma$. Furthermore, by the maximality of $\gamma$, this embedding is essential. Therefore, by the lemma mentioned above, either $\mathbf{A} / \theta$ or $\mathbf{A} / \delta$ is trivial. But $\theta \nsucceq[1,1]$, so we must have $\mathbf{A} / \delta$ trivial, which means $\delta=1_{\mathbf{A}}$, and therefore $\bar{g}^{-1}(\gamma)=\eta_{0}$ as desired.

COROLlaRY 5. Let $\mathscr{V}$ be a congruence distributive variety. If $\mathscr{V}$ has AP and $R S$, then $\mathscr{V}$ has $C E P$.

COROLLARY 6. Let $\mathscr{V}$ be a congruence modular variety that is 2 -finite. If $\mathscr{V}$ has $A P$ and $R S$, then $\mathscr{V}$ has $C E P .^{1}$

PROOF. If $\mathscr{V}$ is congruence distributive, the commutator reduces to intersection. Thus $\mathscr{V}$ trivially has $\mathrm{R}$ and the result follows from Theorem 4 . Suppose $\mathscr{V}$ is congruence modular and 2-finite. By Theorem 1, $\mathscr{V}$ has C2. By [2, Theorem 8] $\mathscr{V}$ has R. Therefore, by Theorem $4, \mathscr{V}$ has CEP.

It is well known that an arbitrary variety has enough injectives if and only if it has AP, RS, and CEP. By the above corollaries, it follows that any congruence modular, 2-finite variety has enough injectives if and only if it has AP and RS.

As applications we offer the following corollaries.

COROLlaRY 7. Let $\mathscr{V}$ be a variety of groups with AP and RS. Then $\mathscr{V}$ is abelian.

ProOF. By Theorem 1, $\mathscr{V}$ satisfies C2. It suffices to show that every subdirectly irreducible group in $\mathscr{V}$ is abelian. Let $\mathbf{G}$ be subdirectly irreducible, and let

${ }^{1}$ ADDED IN PROOF. Recently, Keith Kearnes has shown that the assumption in Corollary 6 that $\mathscr{V}$ be 2 -finite can be dropped. Thus, any congruence modular variety with AP and RS has CEP. 
$\mathbf{M}$ be the normal subgroup corresponding to the monolith. Choose any $a \in M-\{1\}$, and let $\mathbf{A}$ be the subgroup generated by $a$. Then $\mathbf{A}$ is abelian and $\mathbf{G}$ is an essential extension of $\mathbf{A}$. Therefore, by [2, Theorem 7], $\mathbf{G}$ is abelian.

REMARKS. In [10, p. 43] H. Neumann states the following theorem: If $\mathscr{V}$ is a variety of groups with AP, then either every finite member of $\mathscr{V}$ is abelian, or else $\mathscr{V}$ is the variety of all groups.

Observe that the argument in Corollary 7 generalizes to any congruence modular variety $\mathscr{V}$ such that

(i) the free algebra on 2 generators is abelian or

(ii) the free algebra on one generator is nontrivial, abelian and has an idempotent element.

COROLLARY 8 . The variety of all squags is not residually small.

ProOF. A squag is a groupoid $\langle S, \cdot\rangle$ satisfying

$$
x \cdot x=x, \quad x \cdot y=y \cdot x, \quad x \cdot(x \cdot y)=y .
$$

Every squag is a quasigroup ("squag" is short for "Steiner quasigroup"), so it follows that the variety of squags is congruence modular.

It is implicit in Bruck [3, Theorem 2.1] that the variety of squags has AP. On the other hand, Quackenbush $[\mathbf{1 1}, 7.6]$ showed that this variety does not have CEP. It is easy to see that the variety is 2 -finite, in fact the free squag on $\{x, y\}$ has 3 elements: $x, y, x \cdot y$. Therefore, by Corollary 6 , the variety of squags is not residually small.

A variety $\mathscr{V}$ is directly representable if it is finitely generated and contains only finitely many finite, directly indecomposable algebras.

COROLLARY 9. (1) Let $\mathscr{V}$ be a directly representable variety with AP. Then $\mathscr{V}$ has $C E P$. In fact, $\mathscr{V}$ is a varietal product $\mathscr{A} \otimes \mathscr{D}$, in which $\mathscr{A}$ is abelian and $\mathscr{D}$ is a discriminator variety.

(2) Let $\mathscr{V}$ be a congruence modular, semi-simple, finitely generated variety with AP. Then $\mathscr{V}=\mathscr{A} \otimes \mathscr{D}$ in which $\mathscr{A}$ is abelian and $\mathscr{D}$ is filtral.

PROOF. By $[8]$, every directly representable variety is congruence permutable (hence modular), satisfies C2 and every subdirectly irreducible member is either simple or abelian. In case (2), semisimplicity implies $\mathrm{C} 2$. Now let $\mathscr{V}$ represent the variety in either case. $\mathscr{V}$ is finitely generated, hence 2 -finite. $\mathscr{V}$ has $\mathrm{C} 2$, hence $\mathrm{C} 1$, hence is residually small (Theorem 0 ). Therefore, by Corollary $6, \mathscr{V}$ has CEP.

Let $\mathscr{A}$ be the subvariety of $\mathscr{V}$ consisting of all abelian algebras, and let $\mathscr{D}$ be the subvariety generated by all simple, non-abelian algebras. Then by $[\mathbf{7}, 7.2]$, $\mathscr{V}=\mathscr{A} \otimes \mathscr{D}$, and $\mathscr{D}$ is a congruence distributive variety, semisimple and with CEP. Therefore $\mathscr{D}$ is filtral, proving (2). In case (1), $\mathscr{D}$ is congruence permutable as well, so it is a discriminator variety.

\section{REFERENCES}

1. C. Bergman, On the relationship of AP, RS and CEP in congruence modular varieties, Algebra Universalis 22 (1986), 164-171.

2. __ Another consequence of AP in residually small, congruence modular varieties, Houston $\mathrm{J}$. Math. (to appear). 
3. R. H. Bruck, A survey of binary systems, Springer-Verlag, New York, 1971.

4. S. Burris and H. Sankappanavar, A course in universal algebra, Springer-Verlag, New York, 1981.

5. R. Freese and R. McKenzie, Residually small varieties with modular congruence lattices, Trans. Amer. Math. Soc. 264 (1981), 419-430.

6. _ Commutator theory for congruence modular varieties, London Math. Soc. Lecture Notes Ser. 125, Cambridge Univ. Press, New York, 1987.

7. E. Kiss, Injectivity and related concepts in modular varieties I-II. Bull. Austral. Math. Soc. 32 (1985), 35-53.

8. R. McKenzie, Narrowness implies uniformity, Algebra Universalis 15 (1982), 67-85.

9. R. McKenzie, G. McNulty and W. Taylor, Algebras, lattices, varieties, Volume I, Wadsworth \& Brooks/Cole, Monterey, Calif., 1987.

10. H. Neumann, Varieties of groups, Springer-Verlag, Berlin, 1967.

11. R. W. Quackenbush, Varieties of Steiner loops and Steiner quasigroups, Canad. J. Math. 28, 1187-1198.

12. W. Taylor, Residually small varieties, Algebra Universalis 2 (1972), 33-53.

Department of Mathematics, Iowa State University, Ames, Iowa 50011

Department of MATHEMAtics, UNiversity of CALIFornia, Berkeley, CaliforNIA 94720 